\title{
Relationship between Uric Acid Levels and Diagnostic and Prognostic Outcomes in Acute Heart Failure
}

\author{
Queen Henry-Okafor ${ }^{1}$, Sean P. Collins ${ }^{2}$, Cathy A. Jenkins ${ }^{1}$, Karen F. Miller ${ }^{1}$, David J. Maron ${ }^{1}$, \\ Allen J. Naftilan ${ }^{1}$, Neal Weintraub ${ }^{3}$, Gregory J. Fermann ${ }^{2}$, John McPherson ${ }^{1}$, Santosh Menon ${ }^{4}$, \\ Douglas B. Sawyer ${ }^{1}$ and Alan B. Storrow ${ }^{1, *}$
}

\author{
${ }^{1}$ Department of Cardiovascular Medicine, Department of Emergency Medicine and the Department of Biostatistics, \\ Vanderbilt University School of Medicine, Nashville, TN, USA \\ ${ }^{2}$ Department of Emergency Medicine, University of Cincinnati, OH, USA \\ ${ }^{3}$ Division of Cardiology, Department of Internal Medicine, University of Cincinnati, OH, USA \\ ${ }^{4}$ Department of Cardiovascular Medicine The Ohio Heart \& Vascular Center, The Christ Hospital in Cincinnati, OH, \\ USA
}

\begin{abstract}
Objectives: We evaluated the association of plasma uric acid alone and in combination with b-type natriuretic peptide (BNP) for emergency department (ED) diagnosis and 30-day prognosis in patients evaluated for acute heart failure (AHF).

Methods: We prospectively enrolled 322 adult ED patients with suspected AHF. Wilcoxon rank sum test, multivariable logistic regression and likelihood ratio (LR) tests were used for statistical analyses.

Results: Uric acid's diagnostic utility was poor and failed to show significant associations with 30-day clinical outcomes. Uric acid also did not add significantly to BNP results.

Conclusion: Among ED patients with suspected AHF, uric acid has poor diagnostic and prognostic utility.
\end{abstract}

Keywords: Acute heart failure, uric acid, b-type natriuretic peptide, prognostic outcomes.

\section{INTRODUCTION}

Heart failure affects more than five million Americans with many seeking emergency department (ED) evaluation during an acute episode [1-3]. The absence of an accurate prediction tool to aid ED physicians in early risk stratification of acute heart failure (AHF) has led to overestimation of disease severity and overutilization of hospital resources $[4$, 5]. Further, the ED diagnosis disagrees with the diagnosis of cardiologists in $15-20 \%$ of cases, suggesting the need for a more accurate diagnostic tool for ED physicians [6, 7].

Several biomarkers are under investigation as both diagnostic and prognostic adjuncts in AHF. B-type natriuretic peptide (BNP), a protein synthesized and released into the circulation by the myocardium in proportion to mechanical strain, has emerged as a commonly used tool for AHF diagnosis and risk stratification. A high sensitivity has been noted with the use of the manufacturer's recommended cutoff level of $100 \mathrm{pg} / \mathrm{ml}$ for diagnosis of AHF [7]. Despite its util-

*Address correspondence to this author at the Research and Academic Affairs Department of Emergency Medicine Vanderbilt University Medical Center $131321^{\text {st }}$ Avenue, South 703 Oxford House Nashville, TN 372324700 USA; Tel: (615) 936-5934; Fax: (615) 936-4490;

E-mail: alan.b.storrow@vanderbilt.edu ity, some limitations have been identified. BNP levels are elevated in other conditions such as chronic kidney disease, cor pulmonale, pulmonary embolism, left ventricular dysfunction without AHF, and hypotension [8-10]. In addition, BNP levels of $100-500 \mathrm{pg} / \mathrm{ml}$, termed the indeterminate range, present a diagnostic dilemma.

Given the natriuretic peptide limitations, $[9,11,12]$ there arises a need for another biomarker that could improve both its diagnosis and prognosis in heart failure. Uric acid is an interesting biomarker with possible synergy to BNP. Uric acid is a product of the activity of xanthine oxidase and is a final product of purine metabolism [13, 14]. The action of xanthine oxidase has been known to increase during ischemia and heart failure, [15] and hyperuricemia is a common finding in patients with chronic heart failure $[16,17]$. Unlike many novel cardiac biomarkers, the uric acid assay is established, accessible, and inexpensive. Importantly, previous studies suggest uric acid may be useful for AHF diagnosis and risk stratification. However, its use in an ED cohort of patients with signs and symptoms of AHF, in combination with BNP, has not been extensively investigated. Our study aims were two-fold: (1) to assess the association of uric acid with diagnostic and 30-day prognostic outcomes and (2) to assess whether uric acid assisted BNP in diagnostic and 
prognostic outcomes for BNP overall and BNP restricted to the indeterminate zone.

\section{METHODS}

The STRATIFY Study is an ongoing three-center prospective cohort study of adult patients presenting to the ED with signs or symptoms of AHF whose goal is to develop a risk stratification tool to guide ED physicians in disposition decisions. Institutional review boards approved all procedures involved in the STRATIFY study as well as this secondary analysis. Research subjects were recruited per convenience methodology.

\section{Setting and Selection of Participants}

The STRATIFY study population was drawn from consenting patients $\geq 18$ years of age who presented to the ED of three tertiary hospitals (all in the United States) with signs or symptoms of AHF. Specifically, we recruited adult patients who met modified Framingham criteria for AHF (Table 1) and provided written informed consent.

\section{Modified Framingham Criteria for Heart Failure}

The Framingham criteria, reported in 1971, comprise one of the tools to predict heart failure [18-20]. This tool uses "major" and "minor" criteria based on history, physical exam and ancillary tests to diagnose AHF. These are accepted criteria for establishing an etiology of dyspnea before definitive studies have been performed. We used these Criteria to screen ED patients for possible AHF for inclusion in the study. The STRATIFY study targeted an AHF prevalence of $50 \%$. The Framingham criteria were modified by us for ED use by excluding the following: circulation time, vital capacity, weight loss in response to treatment and autopsy findings. These parameters are not routinely available or used in the ED to diagnose AHF.

\section{Data Collection and Processing}

At the time of study entry, ED physicians completed an interview form based on initial patient assessment indicating whether the patient was a candidate. Qualified candidates were approached by trained study coordinators who explained the study purpose and protocol, and obtained written informed consent. Study variables were collected within three hours of the first physician evaluation. Subjects completed questionnaires detailing clinical history such as dyspnea, comorbidities, medication, and social history. The information was later verified using clinical medical records. Information on potential confounding variables such as body weight, history of gout or allopurinol use, and serum creatinine levels were collected from subjects' medical records. The study assistant also collected chest x-ray data based on the final reading available in the medical record by the attending radiologist. All data extracted from the medical record was collected by the study assistants using standardized data abstraction sheets. They were blinded to uric acid values and the outcomes of interest.

Venous blood samples were obtained at enrollment and stored at $-80^{\circ} \mathrm{C}$ until time of testing. Clinical data and blood samples were collected at a single time point. Plasma urate was measured by the central clinical chemistry laboratory using a colorimetric enzymatic in-vitro test processed on the Beckman Coulter ${ }^{\circledR}$ DXC 800 (Beckmann Coulter, Inc., Fullerton, CA). The normal range $(2.0-7.0 \mathrm{pg} / \mathrm{ml})$ for uric acid using the DXC instrument has been validated in-house with healthy patient samples, and was determined to be age non-specific. BNP was measured by the Vanderbilt University Laboratory Services using the Triage B-Type Natriuretic Peptide Test (Biosite Diagnostics Inc., San Diego, California). The triage BNP Test is a fluorescence immunoassay for the quantitative determination of BNP in whole blood and plasma specimens. The reference range for normal values was $<100 \mathrm{pg} / \mathrm{ml}$.

\section{Outcome Measures}

Outcome measures were AHF status (yes or no) and 30day adverse events. The criterion standard for determining AHF status was a medical record review by an expert cardiology oversight group (COG) comprised of three practicing cardiologists on the academic faculty at Vanderbilt University. Cardiology overread is the standard method used to

Table 1. Framingham Criteria for Congestive Heart Failure (CHF)*

\begin{tabular}{|c|c|}
\hline Major Criteria & Minor Criteria \\
\hline Paroxysmal nocturnal dyspnea & Bilateral ankle edema \\
\hline Neck vein distention & Nocturnal cough \\
\hline Rales & Dyspnea on ordinary exertion \\
\hline Radiographic cardiomegaly (increasing heart size on chest radiography) & Hepatomegaly \\
\hline Acute pulmonary edema & Pleural effusion \\
\hline S3 gallop & Decrease in vital capacity by one third from maximum recorded \\
\hline Increased central venous pressure ( $>16 \mathrm{~cm} \mathrm{H} 2 \mathrm{O}$ at right atrium) & Tachycardia (heart rate $>120$ beats/min.) \\
\hline \multicolumn{2}{|l|}{ Hepatojugular reflux } \\
\hline Weight loss $>4.5 \mathrm{~kg}$ in 5 days in response to treatment & \\
\hline
\end{tabular}


determine heart failure presence $[6,7]$. The COG was given all patient records and test results from the index stay as well as follow-up medical records, if any. They were blinded to uric acid results and to each other's diagnosis. The COG was asked to assess whether AHF was the primary diagnosis responsible for the patients' symptoms, present but not primary, or not present in the ED, while blinded to uric acid levels. A consensus was reached if 2 of the cardiologists agreed on the diagnosis. If all 3 disagreed by placing the subjects in 3 distinct groups, then a $4^{\text {th }}$ cardiologist adjudicated the diagnosis. At both 5 and 30 days, study coordinators performed follow-up interviews to document adverse events. These occurred via telephone for discharged subjects or by face-to-face interviews for subjects who were still hospitalized. The events were verified against a subject's medical record by a registered nurse. Only subjects with complete data were included in the analysis.

A 30-day (days 1-30, originally collected as days 1-5 and days 6-30) event was defined as follows: hospitalization for five or more days, ED/hospital readmission, major cardiac events, or death. Major cardiac events were defined as acute coronary syndromes (ACS), cardiac resynchronization therapy (CRT), insertion of implantable cardioverter defibrillator (ICD), emergent renal dialysis, endotracheal intubation, coronary artery bypass graft (CABG), percutaneous coronary intervention (PCI), cardiac transplantation, mechanical cardiac support, palliative hospice or death. Separate analyses were done for outcomes that included all-cause readmission and readmission restricted to AHF as defined by the ED or hospital discharge diagnosis.

\section{Data Analysis}

Statistical analyses were performed using the statistical programming language R, version 2.11.1 [21] and Stata Ver- sion 11.1 [22]. Descriptive statistics on demographic and outcome variables were presented as median (interquartile range, IQR) or frequency (\%), as appropriate. This study was guided by two main objectives.

The primary aim was to determine if uric acid is associated with AHF diagnosis and prognosis. The diagnostic utility of uric acid was assessed using the area under the curve (AUC) for the receiver operating characteristic (ROC) curve. The association between uric acid and the stated outcomes was addressed using both unadjusted and adjusted analyses. Unadjusted tests of association were conducted for continuous uric acid with the outcomes of interest using the nonparametric Wilcoxon Rank Sum test. Unadjusted tests of association were conducted for dichotomized ( $\leq 7.0$ vs $>7.0$ $\mathrm{mg} / \mathrm{dl}$ ) uric acid with the outcomes of interest using the Pearson's chi-square test of association. Multivariable logistic regression models were fit to assess the association of uric acid (continuous and dichotomous) with each of the outcomes adjusting for the following potential confounders selected a priori: history of gout, renal status as determined by the glomerular filtration rate (GFR), and obesity defined as BMI [Ibs/in $\left.{ }^{2}\right]$. The Modification of Diet in Renal Disease (MDRD) study equation and GFR calculator for adults were used for estimating the GFR $\left(\mathrm{mL} / \mathrm{min} / 1.73 \mathrm{~m}^{2}\right)$ from serum creatinine as a measure of renal status [23, 24]. History of gout or documented use of allopurinol was determined through a review of the subject's electronic medical record. Overfitting of the models was avoided by limiting to one covariate for every 10 outcome events.

The second specific aim assessed whether uric acid added useful information in conjunction with BNP in diagnosing AHF or predicting 30 -day outcomes. We assessed the diagnostic utility of uric acid in conjunction with BNP by testing the equality of AUCs for the "full" and "reduced"

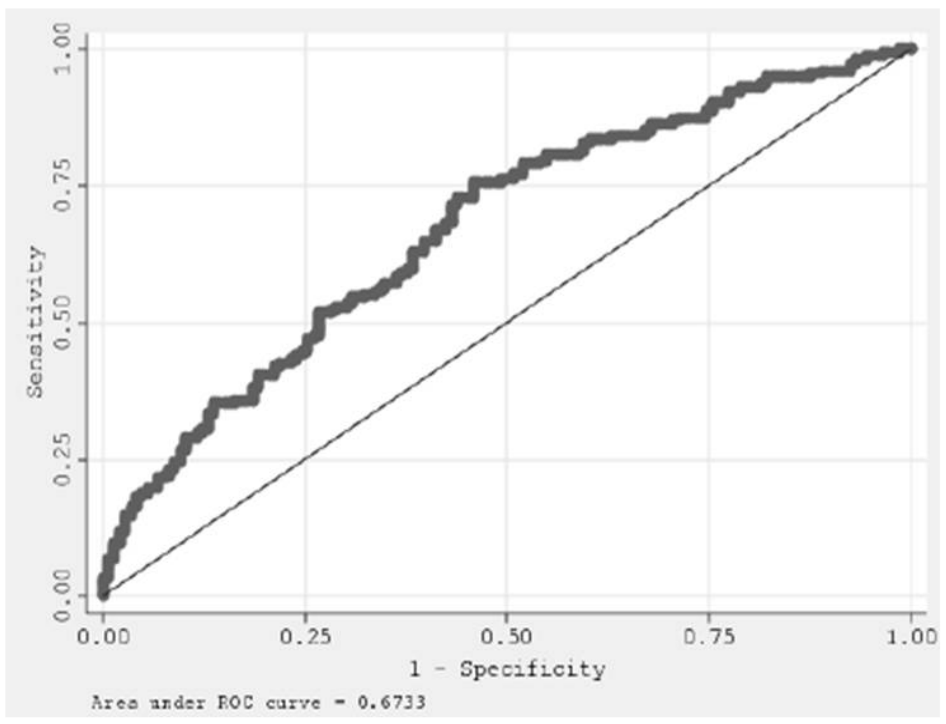

Fig. (1). Area under the receiver operating characteristic curve for the diagnostic utility of uric acid for acute heart failure is 0.67 (98\% $\mathrm{CI}=0.59,0.73)$. 
models using the Pearson's chi-square test of association [25]. This analysis was repeated on the subset of subjects with BNP levels within the $100-500 \mathrm{pg} / \mathrm{ml}$ range to address whether uric acid added useful information to BNP in AHF diagnosis or in predicting 30-day outcomes when BNP levels fell in the indeterminate zone. No adjustments were made for multiple comparisons.

We further assessed if there was a synergistic effect between uric acid and BNP in the diagnosis or prediction of the stated outcomes. To address these, logistic regression models were fit for the various outcomes with BNP, uric acid, race, gender, and chest $\mathrm{x}$-ray status as predictors. A chest $\mathrm{x}$-ray was deemed positive if one of the following conditions was present: pulmonary edema, pleural effusion, interstitial edema, alveolar edema, or vascular congestion. Likelihood ratio (LR) tests were used to assess whether uric acid improves upon BNP in predicting the outcome by comparing the "full" logistic regression model with a "reduced" model excluding uric acid. If uric acid added useful information to the model, then the model was refit with an interaction term between BNP and uric acid to assess whether the relationship between them was synergistic.

\section{RESULTS}

\section{Characteristics of Study Subjects}

Complete records for uric acid, BNP, COG diagnosis, and 30-day outcomes were available in 322 subjects. The median age of the subjects was $64(\mathrm{IQR}=52,75)$, and 182 $(57 \%)$ of the subjects were male (Table 2). A total of 176 subjects were judged to have AHF by our expert panel. Median uric acid was $7.0 \mathrm{mg} / \mathrm{dl}(\mathrm{IQR}=5.5,8.6)$. Subjects with AHF had higher median uric acid levels than those without AHF $(7.5 \mathrm{pg} / \mathrm{ml} ; \quad \mathrm{IQR}=6.2,9.12$ versus $6.15 \mathrm{pg} / \mathrm{ml}$; $\mathrm{IQR}=4.92,7.8$ ). Fig. (1) suggests uric acid has poor diagnostic utility after adjusting for history of gout, GFR, and BMI (AUC $=0.67 ; 98 \% \mathrm{CI}=0.60,0.74)$. After adjusting for history of gout, renal status and BMI (Table 4) uric acid was found to be significantly associated with AHF diagnosis $(\mathrm{OR}=1.29$; $98 \% \mathrm{CI}=1.12,1.48 ; \mathrm{p}=<0.0001)$.

Table 3 details the frequency of the components of the adverse outcomes of interest. Of the 322 subjects, $163 \mathrm{had}$ a 30-day event in which recidivism was for any cause and 132 had a 30-day event where recidivism was restricted to AHF only. The mortality rate among the AHF subjects was small.

Table 2. Demographics, Medical History, and Laboratory Values*

\begin{tabular}{|c|c|c|}
\hline Variables & n (\%) & M (IQR) \\
\hline \multicolumn{3}{|l|}{ Sex } \\
\hline Female & $140(43)$ & \\
\hline Male & $182(57)$ & \\
\hline \multicolumn{3}{|l|}{ Race } \\
\hline Caucasian (non-Hispanic) & $233(72)$ & \\
\hline Hispanic & $1(0)$ & \\
\hline Other & $1(0)$ & \\
\hline \multicolumn{3}{|l|}{ History of gout } \\
\hline No & $189(59)$ & \\
\hline Yes & $129(41)$ & \\
\hline \multicolumn{3}{|l|}{ Uric acid (dichotomized) } \\
\hline$\leq 7.0 \mathrm{mg} / \mathrm{dl}$ & $164(51)$ & \\
\hline$>7.0 \mathrm{mg} / \mathrm{dl}$ & $158(49)$ & \\
\hline Uric acid & & $7.0(5.5,8.6)$ \\
\hline GFR & & $51(32,73)$ \\
\hline BMI & & $30(25,37)$ \\
\hline
\end{tabular}

${ }^{*} \mathrm{~N}=322 ; \uparrow \mathrm{N}=318$. M, Median; IQR, Interquartile range; GFR, glomerular filtration rate; BMI, body mass index; BNP, B- natriuretic peptide. 
Table 3. Distribution of 30-Day AHF Outcomes for All Subjects and for Subjects with BNP Levels of 100-500 pg/ml

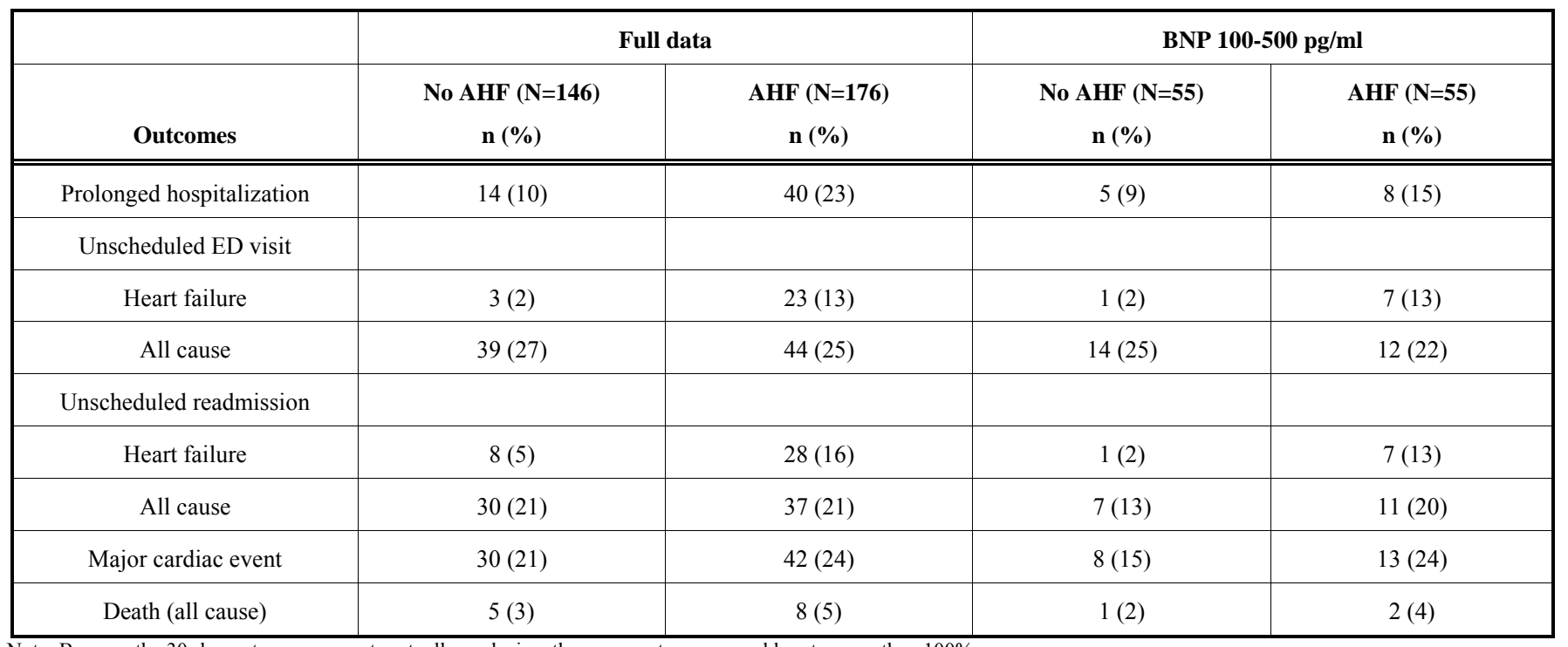

Note: Because the 30-day outcomes are not mutually exclusive, these percentages may add up to more than $100 \%$.

AHF, acute heart failure; BNP, B- natriuretic peptide; ED, emergency department

Table 4. Results from Logistic Regressions for Diagnosis and 30-Day Outcomes Adjusting Continuous Uric Acid for History of Gout, Renal Status as Measured by GFR, and BMI

\begin{tabular}{|c|c|c|c|c|}
\hline Outcome & Covariate & OR & $98 \%$ CI & p Value \\
\hline \multirow[t]{4}{*}{ COG diagnosis (dichotomized) } & Uric acid $\dagger$ & 1.29 & $1.12,1.48$ & $<0.0001$ \\
\hline & History of gout & 1.35 & $0.62,2.95$ & 0.37 \\
\hline & GFR $\dagger$ & 1.00 & $0.98,1.01$ & 0.32 \\
\hline & $\mathrm{BMI} \dagger$ & 1.01 & $0.98,1.03$ & 0.55 \\
\hline \multirow{2}{*}{ 30-day event (all-cause readmission) } & GFR $\dagger$ & 0.99 & $0.98,1.00$ & 0.10 \\
\hline & $\mathrm{BMI} \dagger$ & 1.00 & $0.97,1.02$ & 0.66 \\
\hline \multirow[t]{2}{*}{ 30-day event (readmission for HF only) } & Uric acid $\dagger$ & 1.07 & $0.95,1.20$ & 0.20 \\
\hline & History of gout & 1.43 & $0.69,2.98$ & 0.25 \\
\hline
\end{tabular}

†per one unit. OR, odds ratio; CI, confidence interval; COG, cardiology oversight group; GFR, glomerular filtration rate; BMI, body mass index; HF, heart failure

The majority of the events were divided up similarly among ED return visits, AHF admissions to the hospital, and major cardiac events. We failed to find statistically significant associations between the 30-day outcomes and uric acid except for marginally significant results for the unadjusted analysis of the 30-day outcome in which recidivism was restricted to AHF and uric acid was dichotomized $(p=0.02)$.

Table 5 presents results from the likelihood ratio tests assessing whether uric acid adds value to BNP in both diagnosis and prognosis of AHF. Likelihood ratio tests indicated the addition of uric acid helped BNP in AHF diagnosis as determined by our expert panel after controlling for race, sex, and positive chest $x$-ray $(\mathrm{LR}=17.5, \mathrm{p}<0.0001)$. However, the interaction model suggests BNP and uric acid do not have a synergistic effect in predicting AHF $(\mathrm{p}=0.67)$.
Uric acid failed to show diagnostic utility in conjunction with BNP when comparing AUCs $(\mathrm{AUC}=0.78 ; 98 \% \mathrm{CI}=$ $0.72,0.84 ; \mathrm{p}=0.90$ for both full and reduced models).

Of the 110 subjects with BNP levels between 100-500 $\mathrm{pg} / \mathrm{ml}$, half of them were considered to have AHF by our expert panel. Of these, 13 subjects had major cardiac events while 12 had repeat visit to the ED for all-cause within a 30day period. Eight subjects had prolonged hospitalization and seven had return visits to the ED as well as rehospitalization for AHF (Table 3). Results from likelihood ratio tests in this subset of subjects were similar to the full cohort and suggest uric acid did not add value to BNP in determining 30-day adverse events (Table 5). Further, the data showed that while the addition of uric acid significantly improved the fit of the model for AHF diagnosis $(\mathrm{LR}=17.5 ; \mathrm{p}<0.0001$ and 7.03; 
Table 5. Likelihood Ratio Tests Assessing Additive Value of Uric Acid to BNP

\begin{tabular}{|c|c|c|c|c|c|}
\hline Outcomes & Model & \multicolumn{2}{|c|}{ Full data } & \multicolumn{2}{|c|}{ BNP $100-500 \mathrm{pg} / \mathrm{ml}$} \\
\hline AHF present & $\mathrm{w} /$ interaction & 0.18 & 0.67 & 0.56 & 0.45 \\
\hline 30-day (AHF readmission) & Main effects & 0.76 & 0.38 & 0.83 & 0.36 \\
\hline \multirow[t]{2}{*}{ 30-day (all cause AHF readmission) } & Main effects & 0.02 & 0.90 & 0.002 & 0.97 \\
\hline & $\mathrm{w} /$ interaction & & & & \\
\hline
\end{tabular}

$\mathrm{p}=0.01$, respectively), this relationship was not synergistic $(\mathrm{LR}=0.56 ; \mathrm{p}=0.45)$.

\section{DISCUSSION}

Our results indicated that subjects with a confirmed diagnosis of AHF tended to have higher uric acid levels. While uric acid had overall poor diagnostic utility for AHF, after adjusting for known confounders, uric acid was found to be significantly associated with AHF diagnosis. Unfortunately uric acid was not predictive of 30-day adverse events, nor did it assist BNP in AHF diagnosis or 30-day prognosis.

Although we noted marginally significant results in the unadjusted analysis for the 30-day outcome in which recidivism was restricted to AHF and uric acid was dichotomized, this association did not persist when adjusting for history of gout, GFR, and BMI. In addition, we failed to find that uric acid significantly aided BNP in predicting 30 -day outcomes. The value of uric acid as a diagnostic tool in the ED with prognostic significance requires further investigation. The use of allopurinol for the clinical management of gout is well known, but its impact on symptoms and outcomes in AHF is not. Thanassoulis and colleagues [26] observed improved outcomes among heart failure patients who used allopurinol for treatment of gout. Evidence supporting the clinical use of allopurinol for heart failure is yet to be validated in randomized clinical trials. Screening for hyperuricemia in high risk patients needs further exploration $[14,20]$.

Diuretic use, increased urate production, and diminished renal function often account for higher uric acid levels. Current literature has reported an association between components of the metabolic syndrome such as obesity, hypertension, dyslipidemia, or insulin resistance and renal urate excretion. Although our data failed to show any significant association, the role of the presence of metabolic syndrome in urate excretion is well documented [27, 28].

Our findings are limited by a moderate sample size and the short follow-up period for measuring outcomes. Also, since the majority of patients were white or African American, our findings may not be applicable to those of other races. Moreover, patients enrolled in the STRATIFY study had signs or symptoms of AHF based on the Framingham criteria, which were ideally developed for a longitudinal diagnosis of heart failure. This may introduce bias into patient selection and generalizability of results. Our study is also potentially limited by our use of a cardiology overread. Although this is a standard and accepted method to determine heart failure presence, it is not perfect. The cardiologists were not blinded to BNP results, potentially introducing bias favoring BNP's diagnostic utility. Lastly, subjects' past medical history of gout or allopurinol use were not part of the prospective assessment form. They were collected retrospectively and may have been underestimated.

\section{CONCLUSION}

While our data suggest that uric acid levels are raised in ED patients with AHF, its diagnostic and prognostic utility, whether alone or in combination with BNP, is poor.

\section{CONFLICT OF INTEREST}

None declared.

\section{ACKNOWLEDGEMENTS}

This study was supported by the National Institute for Health: R01 HL088459 (Storrow), K23HL085387 (Collins) and the ARRA R01 HL088459-0S2.

Special thanks to the Clinical Trials Associates who helped with subject recruitment and data entry. We are thankful for data management support provided by Vanderbilt University Research Electronic Data CAPture (REDCap): NIH UL RR024975 (VUMC REDCap).

\section{REFERENCES}

[1] Weintraub WS, Cole J, Tooley JF. Cost and cost-effectiveness studies in heart failure research. Am Heart J 2002; 143(4): 565-76.

[2] Rosamond W, Flegal K, Furie K, et al. Heart disease and stroke statistics--2008 update: a report from the American Heart Association Statistics Committee and Stroke Statistics Subcommittee. Circulation 2008; 29;117(4):e25-146.

[3] Rosamond W, Flegal K, Friday G, et al. Heart disease and stroke statistics--2007 update: a report from the American Heart Association Statistics Committee and Stroke Statistics Subcommittee. Circulation 2007; 115(5): e69-171.

[4] Neily JB, Toto KH, Gardner EB, et al. Potential contributing factors to noncompliance with dietary sodium restriction in patients with heart failure. Am Heart J 2002;143(1): 29-33.

[5] Rame JE, Sheffield MA, Dries DL, et al. Outcomes after emergency department discharge with a primary diagnosis of heart failure. Am Heart J 2001; 142(4): 714-9. 
[6] Collins SP, Peacock WF, Lindsell CJ, et al. S3 detection as a diagnostic and prognostic aid in emergency department patients with acute dyspnea. Ann Emerg Med 2009; 53(6): 748-57.

[7] Maisel AS, Krishnaswamy P, Nowak RM, et al. Rapid measurement of B-type natriuretic peptide in the emergency diagnosis of heart failure. N Engl J Med 2002; 347(3):161-7.

[8] Mak GS, DeMaria A, Clopton P, Maisel AS. Utility of Bnatriuretic peptide in the evaluation of left ventricular diastolic function: comparison with tissue Doppler imaging recordings. Am Heart J 2004; 148(5): 895-902.

[9] Maisel AS, Clopton P, Krishnaswamy P, et al. Impact of age, race, and sex on the ability of B-type natriuretic peptide to aid in the emergency diagnosis of heart failure: results from the Breathing Not Properly (BNP) multinational study. Am Heart J 2004; 147(6): 1078-84.

[10] Wu AH, Harrison A, Maisel AS. Reduced readmission rate for alternating diagnoses of heart failure and pulmonary disease after implementation of B-type natriuretic peptide testing. Eur J Heart Fail 2004; 6(3): 309-12.

[11] Maisel A, Hollander JE, Guss D, et al. Primary results of the Rapid Emergency Department Heart Failure Outpatient Trial (REDHOT). A multicenter study of B-type natriuretic peptide levels, emergency department decision making, and outcomes in patients presenting with shortness of breath. J Am Coll Cardiol 2004; 44(6): 1328-33.

[12] Daniels LB, Bhalla V, Clopton P, et al. B-type natriuretic peptide (BNP) levels and ethnic disparities in perceived severity of heart failure: results from the Rapid Emergency Department Heart Failure Outpatient Trial (REDHOT) multicenter study of BNP levels and emergency department decision making in patients presenting with shortness of breath. J Card Fail 2006;12(4): 281-5.

[13] Bergamini C, Cicoira M, Rossi A, Vassanelli C. Oxidative stress and hyperuricaemia: pathophysiology, clinical relevance, and therapeutic implications in chronic heart failure. Eur J Heart Fail 2009; 11(5): 444-52.

[14] Strazzullo P, Puig JG. Uric acid and oxidative stress: relative impact on cardiovascular risk? Nutr Metab Cardiovasc Dis 2007; 17(6): 409-14.

[15] Ruggiero C, Cherubini A, Ble A, et al. Uric acid and inflammatory markers. Eur Heart J 2006; 27(10): 1174-81.

[16] Doehner W, von Haehling S, Anker SD. Uric acid as a prognostic marker in acute heart failure--new expectations from an old molecule. Eur J Heart Fail 2007; 9(5): 437-9.
[17] Pascual-Figal DA, Hurtado-Martinez JA, Redondo B, Antolinos MJ, Ruiperez JA, Valdes M. Hyperuricaemia and long-term outcome after hospital discharge in acute heart failure patients. Eur J Heart Fail 2007; 9(5): 518-24.

[18] Senni M, Tribouilloy CM, Rodeheffer RJ, et al. Congestive heart failure in the community: a study of all incident cases in Olmsted County, Minnesota, in 1991. Circulation 1998; 98(21): 2282-9.

[19] Ho KK, Anderson KM, Kannel WB, Grossman W, Levy D. Survival after the onset of congestive heart failure in Framingham Heart Study subjects. Circulation 1993; 88(1): 107-15.

[20] McKee PA, Castelli WP, McNamara PM, Kannel WB. The natural history of congestive heart failure: the Framingham study. N Engl J Med 1971; 285(26):1441-6.

[21] R Development Core Team. R: A language and environment for statistical computing. R Foundation for Statistical Computing, editor. Vienna, Austria 2010. Available from http://www.Rproject.org.

[22] StataCorp. Stata Statistical Software: Release 11. College Station, TX: StataCorp LP 2009

[23] Levey AS, Coresh J, Greene T, et al. Expressing the Modification of Diet in Renal Disease Study equation for estimating glomerular filtration rate with standardized serum creatinine values. Clin Chem 2007; 53(4): 766-72.

[24] Stevens LA, Schmid CH, Greene T, et al. Comparative performance of the CKD Epidemiology Collaboration (CKD-EPI) and the Modification of Diet in Renal Disease (MDRD) Study equations for estimating GFR levels above $60 \mathrm{~mL} / \mathrm{min} / 1.73 \mathrm{~m} 2$. Am J Kidney Dis 2010; 56(3): 486-95.

[25] DeLong ER, DeLong DM, Clarke-Pearson DL. Comparing the areas under two or more correlated receiver operating characteristic curves: a nonparametric approach. Biometrics 1988; 44(3): 837-45.

[26] Thanassoulis G, Brophy JM, Richard H, Pilote L. Gout, Allopurinol Use, and Heart Failure Outcomes. Arch Intern Med 2010; 170(15): 1358-64.

[27] Anker SD, Rauchhaus M. Heart failure as a metabolic problem. Eur J Heart Fail. 1999; 1(2): 127-31.

[28] Alimonda AL, Nunez J, Nunez E, et al. Hyperuricemia in acute heart failure. More than a simple spectator? Eur J Intern Med 2009; 20(1): 74-9.

(C) Henry-Okafor et al.; Licensee Bentham Open.

This is an open access article licensed under the terms of the Creative Commons Attribution Non-Commercial License (http://creativecommons.org/licenses/by-nc/3.0/) which permits unrestricted, non-commercial use, distribution and reproduction in any medium, provided the work is properly cited. 